Supporting Information For

\title{
Weak Carbon-Hydrogen-Nitrogen Interactions Affect the Heterocyclic Ligand Bonding Modes in Barium Complexes Containing $\eta^{2}$-Tetrazolato and $\eta^{2}$ - Pentazolato Ligands
}

\author{
Issam Kobrsi, John E. Knox, Mary Jane Heeg, H. Bernhard Schlegel, \\ and Charles H. Winter* \\ Department of Chemistry, Wayne State University, Detroit, Michigan 48202
}

1. Synthetic Procedures, Spectral Data, and Analytical Data for $\mathbf{1}$ and $\mathbf{2}$.

2. Orbital Interactions from Natural Bond Order Analyses of 3-5.

3. Details of the molecular orbital calculations for 3-5.

1. Synthetic Procedures, Spectral Data, and Analytical Data for $\mathbf{1}$ and 2.

General procedure for the preparation of 1 and 2. A Schlenk flask was charged with $2.0 \mathrm{mmol}$ of the appropriate ligand and $1.0 \mathrm{mmol}$ of $\mathrm{Ba}\left[\mathrm{N}\left(\mathrm{SiMe}_{3}\right)_{2}\right]_{2}(\mathrm{THF})_{2}$. Tetrahydrofuran $(20 \mathrm{~mL})$ was added by syringe and the reaction was allowed to stir for 1 $\mathrm{h}$ at room temperature. Next, $1.0 \mathrm{mmol}$ of 18-crown- 6 was added and the suspension was brought to reflux. Dimethylsulfoxide $(\sim 15 \mathrm{~mL})$ was then introduced by syringe slowly until a clear solution was obtained. The hot solution was quickly filtered through a pad of Celite on a coarse glass frit and the filtered solution was allowed to cool to room temperature undisturbed where it was left for $24 \mathrm{~h}$. Complexes $\mathbf{1}$ and $\mathbf{2}$ were isolated as colorless crystals by decanting the solvent, washing with diethyl ether, and then vacuum drying.

[Ba $\left(\boldsymbol{\eta}^{2} \text {-5-dimethylamino-1,2,3,4-tetrazolato }\right)_{2}\left(\boldsymbol{\eta}^{6}\right.$-18-crown-6)] (1). 87\%; mp $260{ }^{\circ} \mathrm{C} \mathrm{dec} ;{ }^{1} \mathrm{H}$ NMR (DMSO- $\left.d_{6}, 22{ }^{\circ} \mathrm{C}, \delta\right) 3.79$ (s, $24 \mathrm{H}, \mathrm{OCH}_{2} \mathrm{CH}_{2} \mathrm{O}$ ), 3.03 (s, 12H, $\left.\mathrm{CH}_{3}\right) ;{ }^{13} \mathrm{C}$ NMR (DMSO-d $\left.6,22{ }^{\circ} \mathrm{C}, \mathrm{ppm}\right) 170.90\left(\mathrm{~s}, \mathrm{CN}_{4}\right), 70.59\left(\mathrm{~s}, \mathrm{OCH}_{2} \mathrm{CH}_{2} \mathrm{O}\right), 40.97$ (s, $-\mathrm{CH}_{3}$ ). Anal. Calcd for $\mathrm{C}_{18} \mathrm{H}_{36} \mathrm{BaN}_{10} \mathrm{O}_{6}: \mathrm{C}, 34.54 ; \mathrm{H}, 5.80 ; \mathrm{N}, 22.38$. Found: $\mathrm{C}$, 34.75; H, 5.87; N, 22.18.

[Ba $\left(\boldsymbol{\eta}^{2} \text {-5-diisopropylamino-1,2,3,4-tetrazolato }\right)_{2}\left(\boldsymbol{\eta}^{\mathbf{6}}\right.$-18-crown-6)] (2). 79\%; mp $292{ }^{\circ} \mathrm{C} \mathrm{dec} ;{ }^{1} \mathrm{H}$ NMR $\left(\mathrm{CDCl}_{3}, 22{ }^{\circ} \mathrm{C}, \delta\right) 4.06$ (septet, J = 6.3 Hz, $\left.4 \mathrm{H},\left(\mathrm{CH}_{3}\right)_{2} \mathrm{CH}\right), 3.81(\mathrm{~s}$,

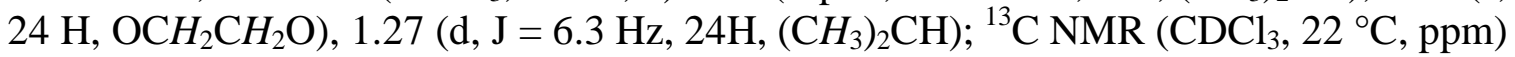
$166.81\left(\mathrm{~s}, \mathrm{CN}_{4}\right), 70.53\left(\mathrm{~s}, \mathrm{OCH}_{2} \mathrm{CH}_{2} \mathrm{O}\right), 47.41\left(\mathrm{~s},\left(\mathrm{CH}_{3}\right)_{2} \mathrm{CH}\right), 21.32\left(\mathrm{~s},\left(\mathrm{CH}_{3}\right)_{2} \mathrm{CH}\right)$. Anal. Calcd for $\mathrm{C}_{26} \mathrm{H}_{52} \mathrm{BaN}_{10} \mathrm{O}_{6}$ : C, 42.31; H, 7.10; N, 18.98. Found: C, 41.20; H, 7.00; N, 18.87.

Reference for the preparation of the aminotetrazoles: Demko, Z.P.; Sharpless, K.B. J. Org. Chem. 2001, 66, 7945. 
2. Orbital Interactions from Natural Bond Order Analyses of 3-5.

Representative NBO fragment orbitals making significant contributions to the stabilization of $\mathbf{3}$.
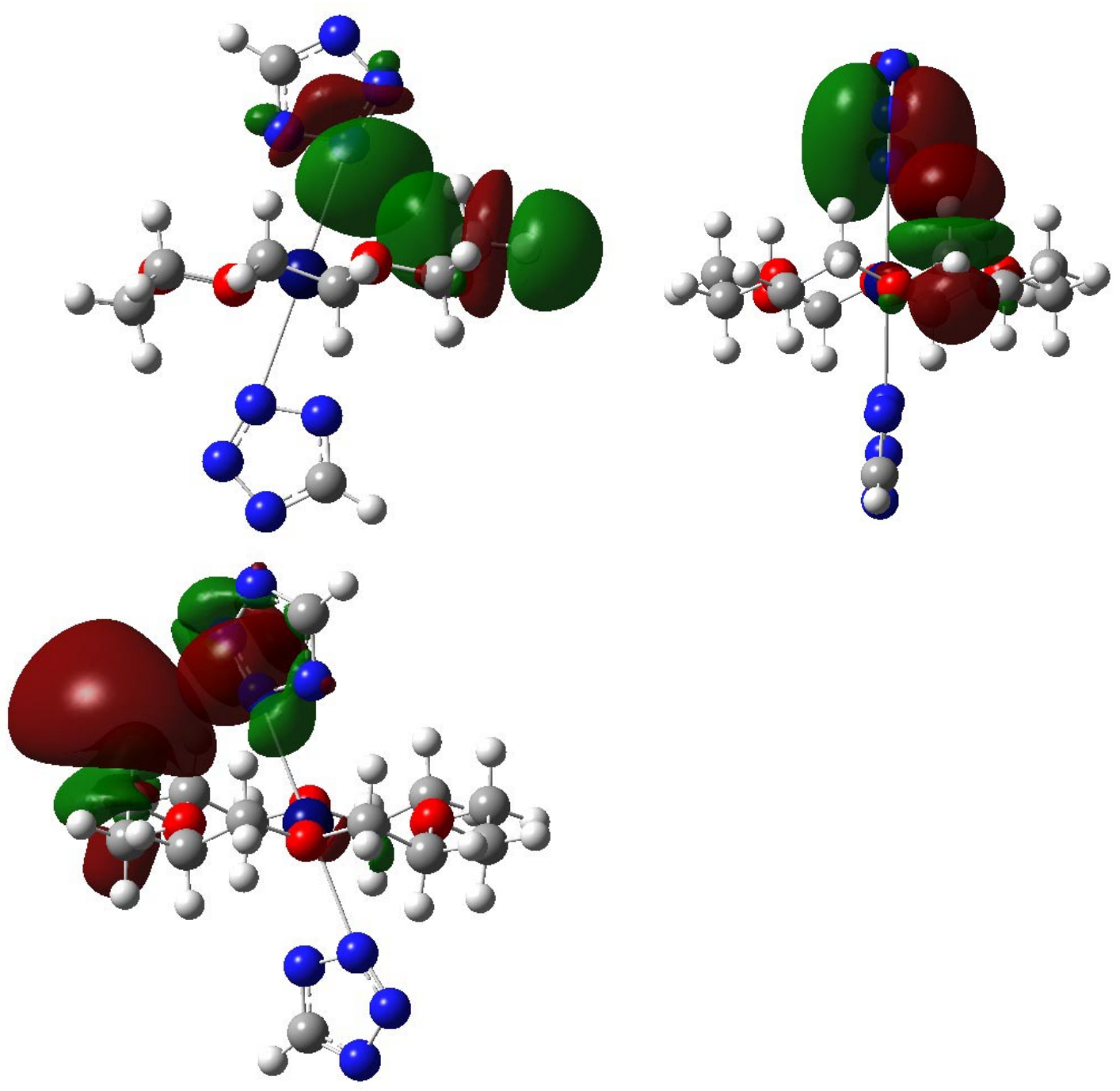
Representative NBO fragment orbital making significant contributions to the stabilization of 4.

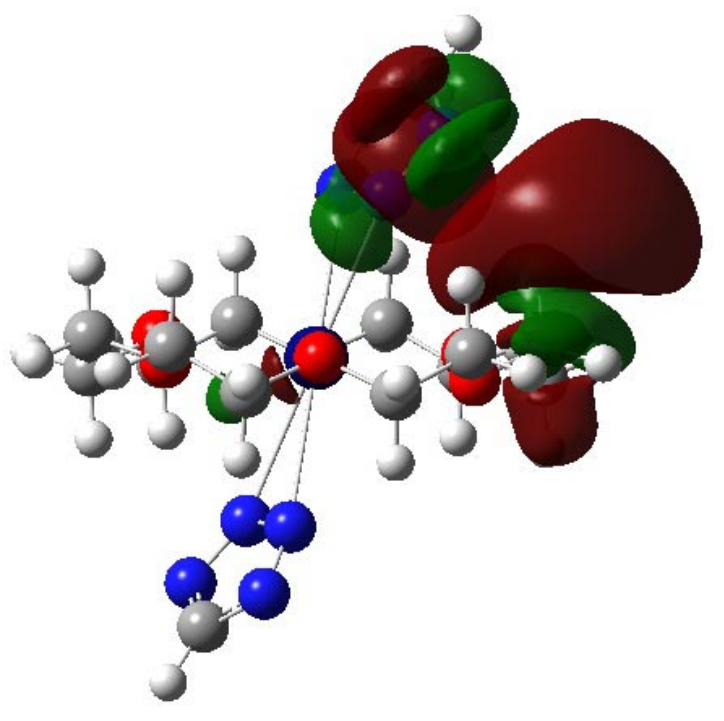

Representative NBO fragment orbitals making significant contributions to the stabilization of $\mathbf{5}$.
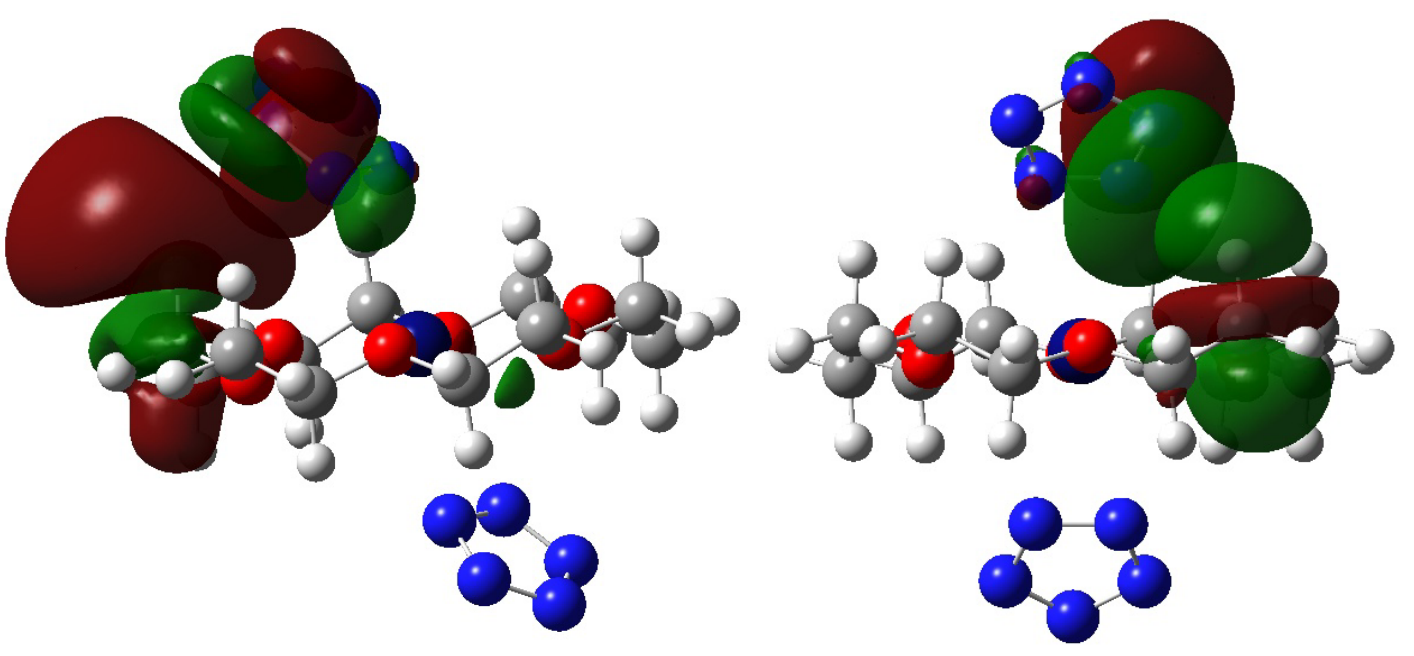

3. Details of the molecular orbital calculations for 3-5.

Optimized Cartesian coordinates of $\mathbf{3}$.

$\begin{array}{lrrr}\mathrm{Ba} & 0.00000000 & 0.00000000 & 0.00000000 \\ \mathrm{O} & -1.30917400 & -1.56105300 & -1.95776300 \\ \mathrm{C} & -1.87225900 & -0.83152000 & -3.05134300 \\ \mathrm{C} & -2.60668500 & 0.38059400 & -2.51564500 \\ \mathrm{O} & -1.66241800 & 1.29843100 & -1.95661100 \\ \mathrm{C} & -2.28213900 & 2.45907300 & -1.39353100 \\ \mathrm{C} & -1.21633900 & 3.36541800 & -0.81223200 \\ \mathrm{O} & -0.63415000 & 2.73619700 & 0.33248200 \\ \mathrm{C} & 0.32115300 & 3.52965600 & 1.03176800 \\ \mathrm{C} & 0.70995200 & 2.80629700 & 2.30674100 \\ \mathrm{~N} & -2.09768500 & -0.80118600 & 1.80079800 \\ \mathrm{~N} & -2.65210600 & -0.03352200 & 0.83880500 \\ \mathrm{~N} & -3.94787100 & 0.05809000 & 1.02240100\end{array}$




$\begin{array}{rrr}-4.28964400 & -0.64417500 & 2.11071200 \\ -3.14022300 & -1.15366500 & 2.56061100 \\ -1.07914600 & -0.52599600 & -3.74567300 \\ -2.58890800 & -1.46655000 & -3.58819200 \\ -3.32848400 & 0.07950100 & -1.74900100 \\ -3.13977600 & 0.86025300 & -3.34821100 \\ -2.99253500 & 2.15806600 & -0.61620400 \\ -2.81518700 & 3.01040200 & -2.18039300 \\ -0.44098200 & 3.57454400 & -1.56011700 \\ -1.68598300 & 4.31148000 & -0.51274300 \\ 1.20632900 & 3.69781100 & 0.40522900 \\ -0.11582200 & 4.50197400 & 1.29602600 \\ 1.42579000 & 3.42672700 & 2.86227400 \\ -0.17509800 & 2.63997200 & 2.93427100 \\ 1.30917400 & 1.56105300 & 1.95776300 \\ 1.87225900 & 0.83152000 & 3.05134300 \\ 2.60668500 & -0.38059400 & 2.51564500 \\ 1.66241800 & -1.29843100 & 1.95661100 \\ 2.28213900 & -2.45907300 & 1.39353100 \\ 1.21633900 & -3.36541800 & 0.81223200 \\ 0.63415000 & -2.73619700 & -0.33248200 \\ -0.32115300 & -3.52965600 & -1.03176800 \\ -0.70995200 & -2.80629700 & -2.30674100 \\ 2.09768500 & 0.80118600 & -1.80079800 \\ 2.65210600 & 0.03352200 & -0.83880500 \\ 3.94787100 & -0.05809000 & -1.02240100 \\ 4.28964400 & 0.64417500 & -2.11071200 \\ 3.14022300 & 1.15366500 & -2.56061100 \\ 1.07914600 & 0.52599600 & 3.74567300 \\ 2.58890800 & 1.46655000 & 3.58819200 \\ 3.32848400 & -0.07950100 & 1.74900100 \\ 3.13977600 & -0.86025300 & 3.34821100 \\ 2.99253500 & -2.15806600 & 0.61620400 \\ 2.81518700 & -3.01040200 & 2.18039300 \\ 0.44098200 & -3.57454400 & 1.56011700 \\ 1.68598300 & -4.31148000 & 0.51274300 \\ -1.20632900 & -3.69781100 & -0.40522900 \\ 0.11582200 & -4.50197400 & -1.29602600 \\ -1.42579000 & -3.42672700 & -2.86227400 \\ 0.17509800 & -2.63997200 & -2.93427100 \\ -3.06132000 & -1.77776500 & 3.43718500 \\ 3.06132000 & 1.77776500 & -3.43718500\end{array}$

\section{Optimized Cartesian coordinates of 4.}

0.00000000

$-0.30868500$

0.21650200

$-0.30470500$

0.35258500

0.00000000

0.35226700

$-0.30509600$

0.21656900

$-0.30867100$

$-3.63269100$

$-2.62112000$

$-2.62226300$

$-3.63476300$

$-4.23298800$

$-1.39944100$

$-0.05253700$

$-1.39117900$

$-0.07181100$

1.44365700

$-0.01258000$

1.44331900

$-0.01311700$

$-1.39148700$

$-0.07265100$

$-1.39942300$
0.00000000

0.69189800

1. 44939100

2. 77849500

3. 49356100

2. 82041000

3.49372300

2. 77851600

1.44953400

0.69190300

1.57979500

0.84000000

0.84480000

1.58763000

2.01898100

0.62338300

1.18947700

2. 74563400

3. 32356400

3.50704000

4.52845800

3.50745600

4.52854000

2. 74528100

3. 32360900

0.62335500
0.00101100

$-3.54143100$

$-2.44646800$

$-2.36979800$

$-1.20654400$

0.00088800

1. 20829700

2. 37143700

2. 44825900

3. 54307700

$-1.11901100$

$-0.66283400$

0.66301000

1. 11203000

$-0.00558600$

$-3.45677500$

$-4.48645900$

$-2.23207200$

$-3.29491900$

$-1.32787600$

$-1.17815400$

1.32982100

1.17980200

2. 23323100

3. 29664400

3.45832200 


$\begin{array}{rrr}-0.05265800 & 1.18936900 & 4.48821100 \\ 0.30868500 & -0.69189800 & -3.54143100 \\ -0.21650200 & -1.44939100 & -2.44646800 \\ 0.30470500 & -2.77849500 & -2.36979800 \\ -0.35258500 & -3.49356100 & -1.20654400 \\ 0.00000000 & -2.82041000 & 0.00088800 \\ -0.35226700 & -3.49372300 & 1.20829700 \\ 0.30509600 & -2.77851600 & 2.37143700 \\ -0.21656900 & -1.44953400 & 2.44825900 \\ 0.30867100 & -0.69190300 & 3.54307700 \\ 3.63269100 & -1.57979500 & -1.11901100 \\ 2.62112000 & -0.84000000 & -0.66283400 \\ 2.62226300 & -0.84480000 & 0.66301000 \\ 3.63476300 & -1.58763000 & 1.11203000 \\ 4.23298800 & -2.01898100 & -0.00558600 \\ 1.39944100 & -0.62338300 & -3.45677500 \\ 0.05253700 & -1.18947700 & -4.48645900 \\ 1.39117900 & -2.74563400 & -2.23207200 \\ 0.07181100 & -3.32356400 & -3.29491900 \\ -1.44365700 & -3.50704000 & -1.32787600 \\ 0.01258000 & -4.52845800 & -1.17815400 \\ -1.44331900 & -3.50745600 & 1.32982100 \\ 0.01311700 & -4.52854000 & 1.17980200 \\ 1.39148700 & -2.74528100 & 2.23323100 \\ 0.07265100 & -3.32360900 & 3.29664400 \\ 1.39942300 & -0.62335500 & 3.45832200 \\ 0.05265800 & -1.18936900 & 4.48821100 \\ 5.10687200 & -2.65191600 & -0.00859000 \\ -5.10687200 & 2.65191600 & -0.00859000\end{array}$

\section{Optimized Cartesian coordinates of $\mathbf{5}$.}

\begin{tabular}{|c|c|c|c|}
\hline $\mathrm{Ba}$ & 0.00000000 & 0.00000000 & 0.00074400 \\
\hline $\mathrm{C}$ & 0.30286000 & 0.69410100 & 3.54968300 \\
\hline 0 & -0.20811700 & 1.43813700 & 2.43817200 \\
\hline C & 0.27777200 & 2.78354000 & 2.37893800 \\
\hline C & -0.37337600 & 3.48540300 & 1.20541500 \\
\hline 0 & 0.00000000 & 2.81420100 & 0.00031600 \\
\hline C & -0.37274700 & 3.48537100 & -1.20498500 \\
\hline C & 0.27885600 & 2.78332100 & -2.37814500 \\
\hline $\mathrm{O}$ & -0.20693100 & 1.43790900 & -2.43719000 \\
\hline $\mathrm{C}$ & 0.30324900 & 0.69392400 & -3.54907200 \\
\hline $\mathrm{N}$ & 3.50868300 & 1.78214400 & 1.07102600 \\
\hline $\mathrm{N}$ & 2.65143800 & 0.86493100 & 0.66611700 \\
\hline $\mathrm{N}$ & 2.65096600 & 0.85811900 & -0.66133000 \\
\hline $\mathrm{N}$ & 3.50780700 & 1.77120700 & -1.07644000 \\
\hline $\mathrm{H}$ & 1.39558800 & 0.63560000 & 3.48638200 \\
\hline $\mathrm{H}$ & 0.02539800 & 1.19616300 & 4.48582100 \\
\hline $\mathrm{H}$ & 1.36763600 & 2.78324900 & 2.26863400 \\
\hline $\mathrm{H}$ & 0.00776200 & 3.31668600 & 3.30025800 \\
\hline $\mathrm{H}$ & -1.46593600 & 3.48498700 & 1.31191900 \\
\hline $\mathrm{H}$ & -0.02078800 & 4.52405400 & 1.17824300 \\
\hline $\mathrm{H}$ & -1.46524900 & 3.48501900 & -1.31203100 \\
\hline $\mathrm{H}$ & -0.02007300 & 4.52399100 & -1.17771500 \\
\hline $\mathrm{H}$ & 1.36871500 & 2.78305400 & -2.26765300 \\
\hline $\mathrm{H}$ & 0.00911600 & 3.31627000 & -3.29965700 \\
\hline $\mathrm{H}$ & 1.39598200 & 0.63488400 & -3.48628900 \\
\hline $\mathrm{H}$ & 0.02565100 & 1.19631300 & -4.48499700 \\
\hline $\mathrm{C}$ & -0.30286000 & -0.69410100 & 3.54968300 \\
\hline 0 & 0.20811700 & -1.43813700 & 2.43817200 \\
\hline $\mathrm{C}$ & -0.27777200 & -2.78354000 & 2.37893800 \\
\hline $\mathrm{C}$ & 0.37337600 & -3.48540300 & 1.20541500 \\
\hline 0 & 0.00000000 & -2.81420100 & 0.00031600 \\
\hline $\mathrm{C}$ & 0.37274700 & -3.48537100 & -1.20498500 \\
\hline $\mathrm{C}$ & -0.27885600 & -2.78332100 & -2.37814500 \\
\hline 0 & 0.20693100 & -1.43790900 & -2.43719000 \\
\hline $\mathrm{C}$ & -0.30324900 & -0.69392400 & -3.54907200 \\
\hline $\mathrm{N}$ & -3.50868300 & -1.78214400 & 1.07102600 \\
\hline $\mathrm{N}$ & -2.65143800 & -0.86493100 & 0.66611700 \\
\hline $\mathrm{N}$ & -2.65096600 & -0.85811900 & -0.66133000 \\
\hline $\mathrm{N}$ & -3.50780700 & -1.77120700 & -1.07644000 \\
\hline
\end{tabular}




\begin{abstract}
$-1.39558800$
$-0.02539800$

$-1.36763600$

$-0.00776200$

1. 46593600

0.02078800

1.46524900

0.02007300

$-1.36871500$

$-0.00911600$

$-1.39598200$

$-0.02565100$

4.03902200

$-4.03902200$
\end{abstract}

$-0.63560000$

$-1.19616300$

$-2.78324900$

$-3.31668600$

$-3.48498700$

$-4.52405400$

$-3.48501900$

$-4.52399100$

$-2.78305400$

$-3.31627000$

$-0.63488400$

$-1.19631300$

2.33956900

$-2.33956900$
3.48638200

4.48582100

2. 26863400

3. 30025800

1. 31191900

1.17824300

$-1.31203100$

$-1.17771500$

$-2.26765300$

$-3.29965700$

$-3.48628900$

$-4.48499700$

$-0.00582000$

$-0.00582000$ 